\title{
A DECISION SuPPort SySTEM FOR TUBERCulosis DIAGNOSABILITY
}

\author{
Navneet Walia ${ }^{1}$, Harsukpreet Singh $^{2}$, Sharad Kumar Tiwari ${ }^{3}$ and Anurag Sharma ${ }^{4}$ \\ ${ }^{1,2,4}$ Department of Electronics and Communication Engineering, CT Institute of \\ Technology and Research (PTU), Jalandhar \\ ${ }^{3}$ Department of Electrical and Instrumentation, Thapar University, Patiala
}

\begin{abstract}
In order to cope with real-world problems more effectively, we tend to design a decision support system for tuberculosis bacterium class identification. In this paper, we are concerned to propose a fuzzy diagnosability approach, which takes value between $\{0,1\}$ and based on observability of events, we formalized the construction of diagnoses that are used to perform diagnosis. In particular, we present a framework of the fuzzy expert system; discuss the suitability of artificial intelligence as a novel soft paradigm and reviews work from the literature for the development of a medical diagnostic system. The newly proposed approach allows us to deal with problems of diagnosability for both crisp and fuzzy value of input data. Accuracy analysis of designed decision support system based on demographic data was done by comparing expert knowledge and system generated response. This basic emblematic approach using fuzzy inference system is presented that describes a technique to forecast the existence of bacterium and provides support platform to pulmonary researchers in identifying the ailment effectively.
\end{abstract}

\section{KEYWORDS}

Expert system, fuzzy diagnosability, rulebased method, MATLAB, Tuberculosis (TB).

\section{INTRODUCTION}

Medical diagnosis of diseases is one of the most foremost important issues in the healthcare unit. The medical industry is one of the new fields, which requires engineering technologies to access uncertain information objectively. With recent developments in medical engineering and other control areas have been achieved by state-of-art intelligent computing techniques ranging from computer-aided diagnosis, computer aided recognition, pattern recognition, bioinformatics, text categorization and intensive care unit [kavita]. Making use of artificial intelligence, information processing, and data mining hold new strategies for approximate inference. Artificial intelligence has witnessed an intensive research interest towards integrated different computing paradigms together including fuzzy logic, artificial neural network, and genetic algorithms. All these methodologies work together and provide flexible information capabilities from one form to another to handle real life ambiguous situations [5]. An emerging class of intelligent machines that could aid in physicians diagnosis is the development of clinical diagnosis decision support system. This clinical system is defined as a computer program designed to assist physicians and health experts in making clinical decision tasks. Clinical decision support system is broadly classified as Knowledge-based clinical decision support system; Non-knowledge based clinical decision support system [16]. 
The knowledge-based decision support system mainly consists of If-then type rules, which are also referred as production rules. The knowledge-based system mainly constitutes three main parts: knowledge base, database rules and inference engine mechanism. Knowledge based system comprises of the database model and fuzzy logic model. The inference engine uses set of rules to combine patient information and to provide output. In particular, fuzzy systems models are useful in situations involving highly complex systems whose behaviour is not well understood and in diagnosing and predicting situations where approximate, but fast solution is required $[16,22]$.

Non knowledge clinical decision support systems are the system that focuses on the usage of artificial intelligence is termed as machine learning algorithm or non-knowledge based clinical decision support system. It is further classified as Neural network (NN) and Genetic algorithm (GA). The structure of the neural network is a mathematical representation of human neural architecture making use of learning and generalization abilities. It consists of a large number of simple, highly interconnected processing elements (artificial neurons) inspired from neuroscience or neurobiology. Each neuron in a layer is interconnected to another neuron in next layer through a weighted interconnection. The neural network makes use of weighted connections and nodes to represent the relationship between symptoms and diagnosis [15, 16]. Genetic algorithm is subclass of an evolutionary algorithm that makes use of biology inspired mechanism, where elements of search space are binary strings (chromosomes) which correspond to a particular solution. GA is a powerful tool for optimization of fuzzy rule-based system and complex problems. These systems are deployed for optimal selection of antecedents and consequents in a fuzzy system. The major weakness of genetic systems is that it usually tends to be, computationally expensive in real systems, premature convergence and slow search speed. This system is appropriate when we do not require the best solution, the only appropriate solution is required [10 11].

Fuzzy set theory, which was proposed by Prof. Lofti Zahed in 1965 [1], makes it possible to define inexact medical entities in more human compressible or natural form. In the field of medicine information available to physicians related to patient and about the medical relationship is characterized by an inherent lack of certainty, incompleteness, and inconsistency. The present work discusses a medical expert system making use of fuzzy logic to identify ailment stage from its prescribed symptoms. Dataset collected from 65 different patients' records which are obtained from a health clinic. Accuracy analysis is calculated using patient record having 9 different attributes which cover demographical data. With expert knowledge fuzzy rules are developed that can be fired during the decision process. This paper introduces the design of knowledge-based medical decision support system for diagnosis of tuberculosis. The proposed system will be equipped with data mining and artificial intelligence techniques such as fuzzy logic techniques in order to become an active distributed medical advisory system. Rule based method using fuzzy logic is implemented to diagnose Mycobacterium tuberculosis bacilli (TB). Detection of mycobacterium tuberculosis organism at initial stages is very important in order to prevent its growth and maintain world's population [22]. According to the studies conducted by World Health organization a third part of world population (1722 million people) are carriers of these bacteria, originating 10 million new cases of active TB worldwide and approximately 3 million death annually [2]. Pulmonary TB is a contagious bacterial infection that involves lungs and can infect other organs or tissues such as a brain, kidneys, bone, and skin. Typical outward indications of pulmonary TB includes a persistent cough, chest pain, hemoptysis, smoke addiction, BCG vaccine, malaises, loss of appetite, occasional fever and reduction in weight [22]. 


\section{REVIEW OF RELATED WORK}

In this section, we will introduce some related works in the field of fuzzy logic. A detailed survey of fuzzy logic techniques is found during this section. There are many works in literature that explains design and implementation of medical experts system.

A novel approach to identify tuberculosis bacteria based on shape and colour was proposed by $\mathbf{M}$. Forero et al. (2004). Designed algorithm technique was based on combined use of invariant shape features together of bacilli with simple thresholding operation on chromatic channels. This methodology is based on segmentation followed by an identification procedure, for which 110 samples of bacilli was analyzed. Usefulness of K-means clustering algorithm techniques was applied to predict classification, accuracy, and sensitivity versus specificity was evaluated using ROC analysis procedure. Further, the author suggested exploring a colour-based edge segmentation technique using derivative operators to all chromatic channels and by using Bayesian decision theory [2]. N. Walia et al. (2015) had presented a systematic approach for design and identification of tuberculosis using fuzzy based decision support system. Their framework briefly explains relation between different input attributes and its symptoms. Author concluded that fuzzy basis dependent expert systems can be used during diagnosis. Further, author suggested that designed system can be extended for construction of other chronic obstructive diseases using hybrid neuro systems [delhi]. An integrated approach for automated detection of early lung cancer and tuberculosis based X-ray image analysis was demonstrated by K. Lee (2006). Various symptoms of the disease and finding nodules were focused during this paper. The proposed technique uses watershed segmentation approach to isolate a lung X-ray image, and then apply a small scanning window to determine whether any pixel is a part of a disease nodule or not. Additionally, various methods used to detect early signs of cancer and tuberculosis was also explained in this work [4]. N. Walia et al. (2015) had clearly explained the working of adaptive neuro fuzzy inference system. Their work comprises of various studies of sugeno and mamdani type system. Layer by layered architecture of hybrid network via aid of artificial intelligence was examined [walia]. A computational intelligent approach for estimation of infectious disease and resource utilization was discussed by E. Papageorgiou et al. (2009). Fuzzy cognitive map based tool was used to represent medical diagnosis system concentrated on pulmonary infections. Due to easy graphical representation approach, the proposed method makes wide use of computer consultation system. Further, FCM can be applied to determine the severity of infection especially in the problem of infectious Pneumonia. The presented system would offer a solution for requirements imposed by the target application, disease symptoms, signs and laboratory tests [6]. Usefulness of fuzzy logic approach to decision support system in medicine was explored by U. Dev et al. (2011). This approach was based on the prognosis of a patient suffering heart failure treated with beta blockers. The developed system is a prototype warning system for clinical problems which is based on the assumption that can be analyzed using simple rules. The proposed technique generates basic rules using fuzzy logic based on expert experience [9]. The decision-making process in real life problems is too complex so soft computing tool can be used to model diagnosis process effectively. A spectrum of soft computing decision-making model to solve a real life complex problem related with medical science was explored by $\mathbf{P}$. Srivasta et al. (2013). The designed network was tested with ECG analysis and the satisfactory factor was measured under a domain of considered inputs [21]. To handle imperfect facts, missing information and decision introduced into a complex system, a novel Intuitionistic fuzzy cognitive map (iFCM) based on theory of Intuitionistic fuzzy sets was explained was M. Arts et al. (2013). This model offers checking and classification techniques to predict human decision model. The proposed system has an extension of FCM to the co-evaluate degree of hesitation; experts may suffer while defining a relation between concepts of FCM. The author demonstrates the effectiveness of FCM with numeric reproducible expels on a process of control and decision support. The simulation studies describe the performance of iFCM for medical decision support 
and the results obtained were significantly better than obtained with conventional FCM model [13]. A dental based expert system called ED (Electronic dentist) was explained by $\mathbf{O}$. Tinuke et al. (2015). Combining artificial intelligence techniques known as ANN and FL, a new system was developed which is a web-based application designed to replace treatment. This system uses coactive Neuro Fuzzy method to diagnose mild dental problems. The simulation was implemented using C\# programming language. The author suggested that designed system reduces the stress involved in the treatment of most medical practitioners [23].

It is inferred from above literature survey on fuzzy logic that they are successfully applied in many medical fields for diagnosing and monitoring of various diseases. In this work, fuzzy rules are applied to determine the stage of tuberculosis.

\section{FUZZY DIAGNOSTIC DECISION SUPPORT SYSTEM}

\subsection{Materials and Method}

Medical expert technology utilizing branch of artificial intelligence has successfully moved from laboratory to real life applications. The fuzzy logic module can be used as a decision-making tool to approximate patient's lung disease by means of fuzzy relationships. In this section, preparing tuberculosis dataset and implementing results using fuzzy inference system for diagnosing the disease of tuberculosis patients are considered. After finding the satisfying degree of similarity conclusion of accuracy, can be obtained based on a dataset of 65 patients collected from government health clinic. Using 9 numbers of the input attributes (symptoms) fuzzy inference system is constructed. The rule-based decision making unit uses expert knowledge to deal with the elementary conjunction of the patient symptom and make an appropriate decision according to the constructed fuzzy rules.

\subsection{DOMAIN ATTRIBUTES OF INPUT VARIABLE}

Input dataset attributes are based on demographic data and clinical finding. In the first group, the cough is categorized into three classes the patient have, ' 0 ' indicates a cough is less than two week, ' 1 ' indicates a cough is between two to three weeks, '2' indicates a cough is more than three weeks. BCG vaccine attribute shows that whether the patient has taken bacillus CalmetteGuerin vaccination or not. Chest pain, Malaises, loss of appetite and loss in weight has binary values. All these parameter has two values, either positive or negative. Smoke addiction parameter indicates a number of cigarettes consumed by a person per day. It consists of three subgroups, ' 0 ' indicates patient is a non-smoker, ' 1 ' indicates patient takes less than eight cigars per day, ' 2 ' indicates patient takes 6 to 10 cigars per day. Fever is classified into three classes, ' 0 ' means normal fever value which is nearly $36.5^{\circ} \mathrm{C}$, ' 1 ' means fever value high, ' 2 ' means sub febrile fever value which exceeded $38.5^{\circ} \mathrm{C}$. Haemoptysis parameter indicates there is coming of blood from respiratory tract of patient while coughing or not. It can be either positive or negative. Table 1 lists all the domain values of input with their data type and data domains. 
Table1. List of input attributes and domain value

\begin{tabular}{|l|l|l|}
\hline Input attribute & \multicolumn{1}{|c|}{ Data type } & Acceptable score \\
\hline Coughing & Integer & $\begin{array}{l}\text { 0 mean }<\text { two } \\
\text { weeks, } 1 \text { mean } \\
\text { between two- } \\
\text { three weeks, } \\
\text { mean }>\text { three } \\
\text { weeks }\end{array}$ \\
\hline BCG & Boolean & Yes $=0$, No $=1$ \\
\hline Chest Pain & Boolean & No $=0$, Yes $=1$ \\
\hline Malaises & Boolean & No $=0$, Yes $=1$ \\
\hline Fever & Integer & $\begin{array}{l}\text { mean normal, 1 } \\
\text { mean high, } \\
\text { mean subfebrile }\end{array}$ \\
\hline Loss of appetite & Boolean & Yes $=0$, No $=1$ \\
\hline Smoke addiction & Integer & $\begin{array}{l}\text { mean none, 1 } \\
\text { mean less than } \\
\text { eight, 2 mean } \\
\text { eight to ten }\end{array}$ \\
\hline Weight loss & Boolean & No $=0$, Yes $=1$ \\
\hline Haemoptysis & Boolean & No $=0$, Yes =1 \\
\hline
\end{tabular}

\subsection{Proposed System ArChitecture}

This section describes the approach adopted in developing the overall fuzzy framework for decision support system. Fuzzy inference system is a computing framework based on concepts of fuzzy set theory, accepts a fuzzy description of patient's symptoms and infers fuzzy relationship accordingly. In order to exploit the fuzzy representation to full, i.e., to achieve higher interpretability, the ability to learn generalization is of great importance. With generalization, we understand in this paper capability to express the state-action relationships as compact as possible. Generalized rules allow more compact rule bases, scalability to higher dimensional spaces, faster inference, and better linguistic interpretability. A fuzzy based decision support system adopts expert's knowledge and knowledge of IF-THEN rules, to implement fuzzy based reasoning $[1,20]$. Thus, fuzzy logic provides an easy way of building an optimal solution with direct guidance from an infeasible region. A membership function associated with a given fuzzy set maps input value to its appropriate membership and its value lies between $(0,1)$. A fuzzy set is a set without a crisp value, it has fuzzy boundaries. Fuzzy set A in universe of discourse $\mathrm{X}$ is defined as set of ordered pair of elements $\mathrm{x}$ in $\mathrm{X}$ as,

$$
\mathrm{A}=\left\{\mathrm{x}, \mu_{\mathrm{A}}(\mathrm{x}), \quad \mathrm{x} € \mathrm{X}\right\}
$$

where $\mu_{\mathrm{A}}(\mathrm{x})$, is called grade membership function of $\mathrm{x}$ in A. all fuzzy set consists of elements having partial membership boundaries [17]. The triangular membership function curve is function having three variables $\mathrm{p}, \mathrm{q}$, and $\mathrm{r}$ in $\mathrm{x}$-axis where $\mathrm{p}, \mathrm{r}$ are called 'feet values' respectively, having membership degree as zero and q are called 'peak value' having membership degree as one. The triangular membership function is represented by using 
equation 1.

$f(x, p, q, r)=\left\{\begin{array}{cl}0 & \text { if } x<=p \\ \frac{x-p}{q-p} & \text { if } p<x \leq q \\ \frac{r-p}{r-q} & \text { if } \mathrm{q} \leq x<r \\ 0 & \text { if } x>=r\end{array}\right\}$

Trapezoidal membership function is represented by using four variables $\mathrm{p}, \mathrm{q}, \mathrm{r}, \mathrm{s}$ in $\mathrm{x}$-axis where $\mathrm{p}$ and s locate 'feet' of trapezoid whose membership degree is zero, parameter $q, r$ locate 'shoulders' whose membership is one represented by using equation 2 .

$f(x, p, q, r, s)=\left\{\begin{array}{cl}0 & \text { if } x<=p \\ \frac{x-p}{q-p} & \text { if } p \leq x<q \\ \frac{s-x}{s-r} & \text { if } r \leq x<s \\ 0 & \text { if } s<=x\end{array}\right\}$

For designing fuzzy inference system, the fuzzy logic toolbox for Matlab has a structure that can easily modify. The fuzzy inference model is the Matlab object that contains information about fuzzy inference system variables such as defining of membership function of input and output variables, rulebase etc. The structure of model is a basically a hierarchy of structures as shown in figure 1. The role of membership function parameters for every input and output variable are confined within the respective universe of discourse and at the same time satisfy the constraint defined to ensure overlapping between the adjacent membership functions.

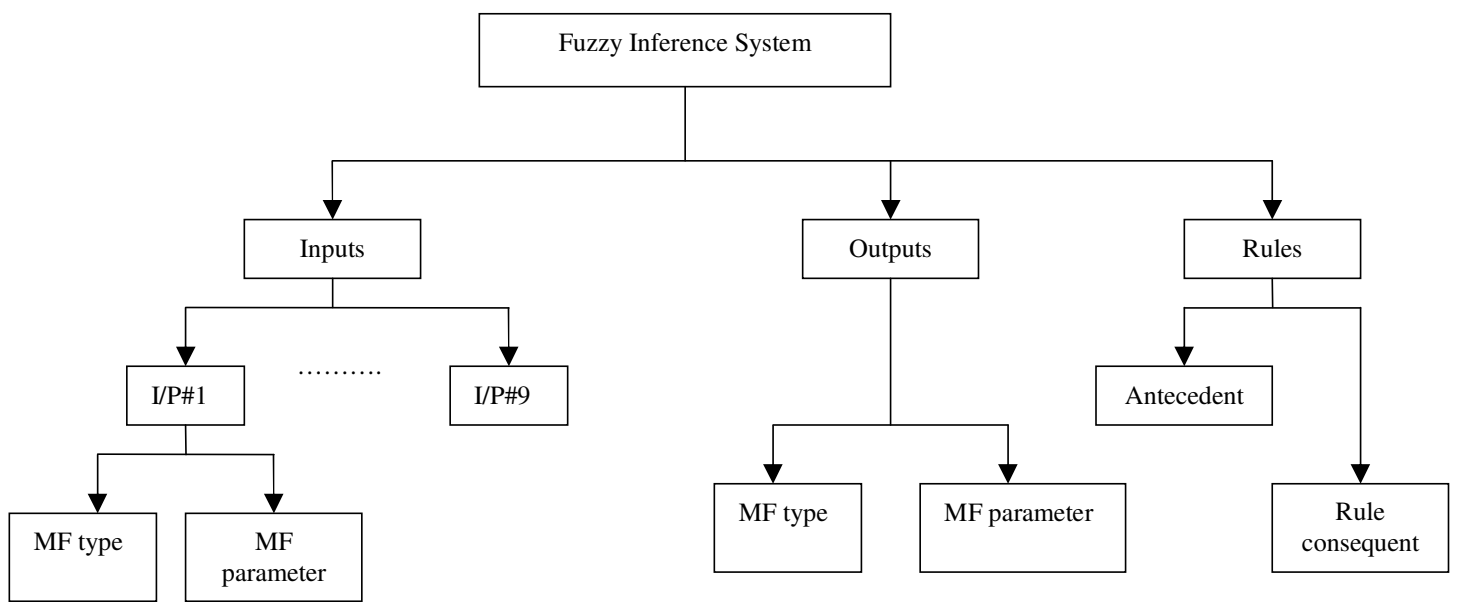

Figure 1. The Fuzzy Inference Structure

In this paper, we propose fuzzy rule-based decision based system implemented for pulmonary physicians, to determine a severity of the ailment. Figure 2 shows proposed system architecture, to assist inexperienced physicians in arriving at final diagnosis of tuberculosis more efficiently and proficiently. 


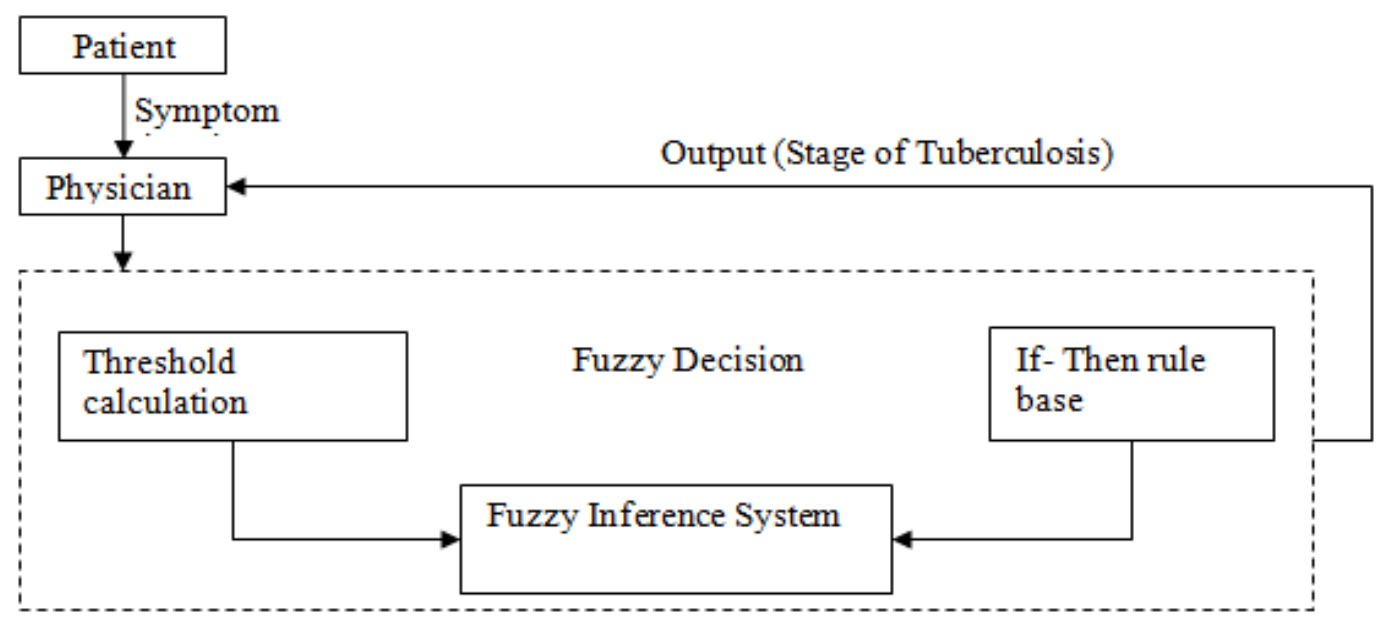

Figure 2. Framework of proposed system architecture

Based on fuzzy set theory and fuzzy rules, a framework of the fuzzy rule-based system is proposed. This method uses the rule base to evaluate precise output values according to the input values. The prediction of patient ailment stage is calculated by fuzzy decision making unit consisting of an algorithm for threshold calculation, inference engine, if-then rule database. The purpose of fuzzification is, depending on the definite membership functions, to renovate the input to the numerical value, such as a maximum of coordinate offset, into the set of fuzzy variables. According to the interpretation of fuzzy linguistic terms, input attributes ranging from 1 to 9 input values uses following terms, Normal (NL), high (HG), low (LW), yes (Y), no (N), severe (SV), extreme (EX). The knowledge base consists of 168 rules, each symptom, and their corresponding condition is based on its weight. The mapping of output is accomplished by an expert knowledge that undergoes defuzzification process in order to compute crisp output stage. Figure 3 the shows the flowchart for diagnosis process.

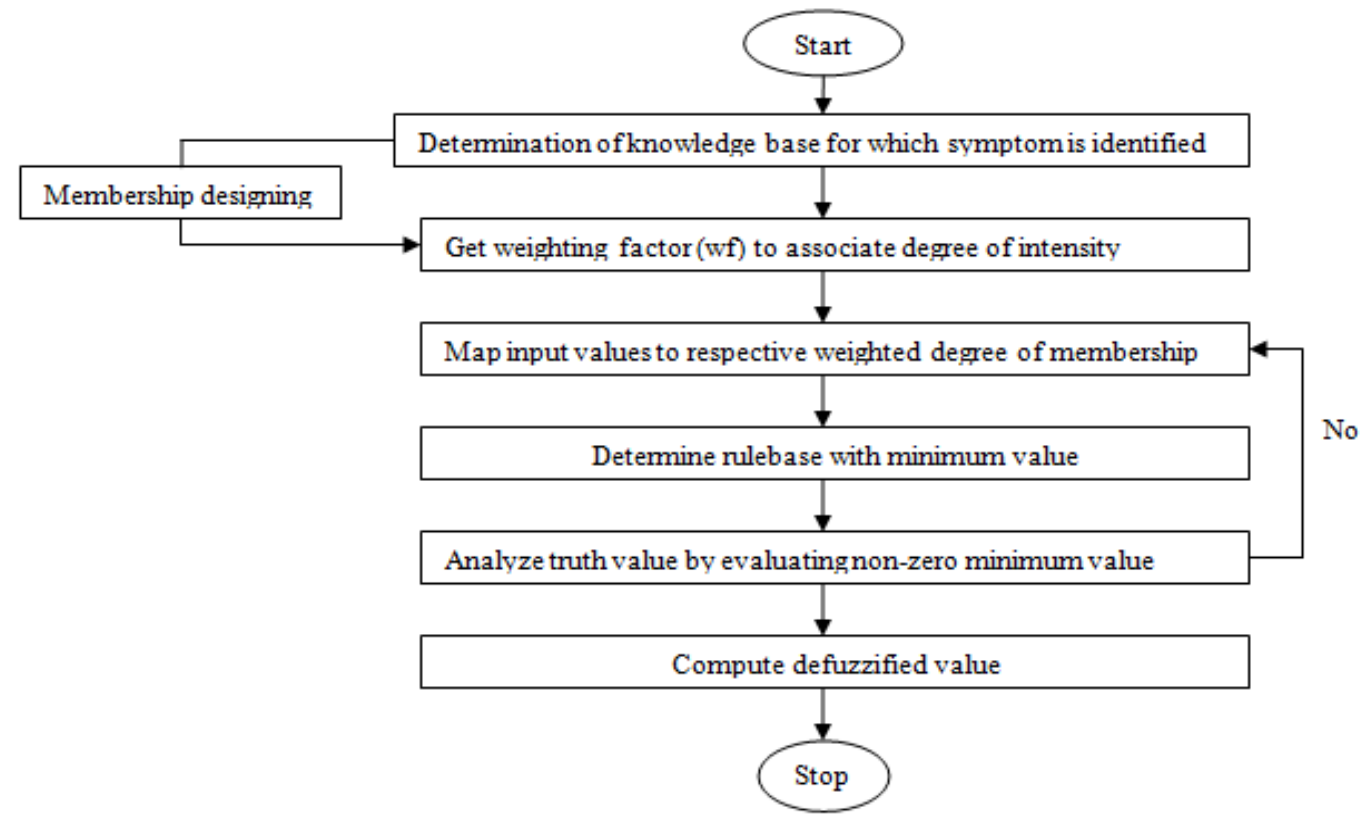

Figure 3. Flowchart for medical diagnosis 


\section{SYSTEM MODELLING AND WORKING}

Fuzzy rules and fuzzy reasoning are the backbones of fuzzy inference system that transforms system inputs (crisp numbers) into fuzzy sets for determination of actual output stage. Fuzzy ifthen rules are production rules including antecedents and consequent parts in the form IF A (antecedent) THEN C (consequent) where $\mathrm{A}$ and $\mathrm{C}$ are specific knowledge related to input and output variables. This paper is simulation based paper and the medical diagnosis is performed on a fuzzy logic model by employing MATLAB 2013b software package developed by Mathworks Inc. The system used to determine the occurrence of tuberculosis and its membership plot as shown in Figure 4-10.

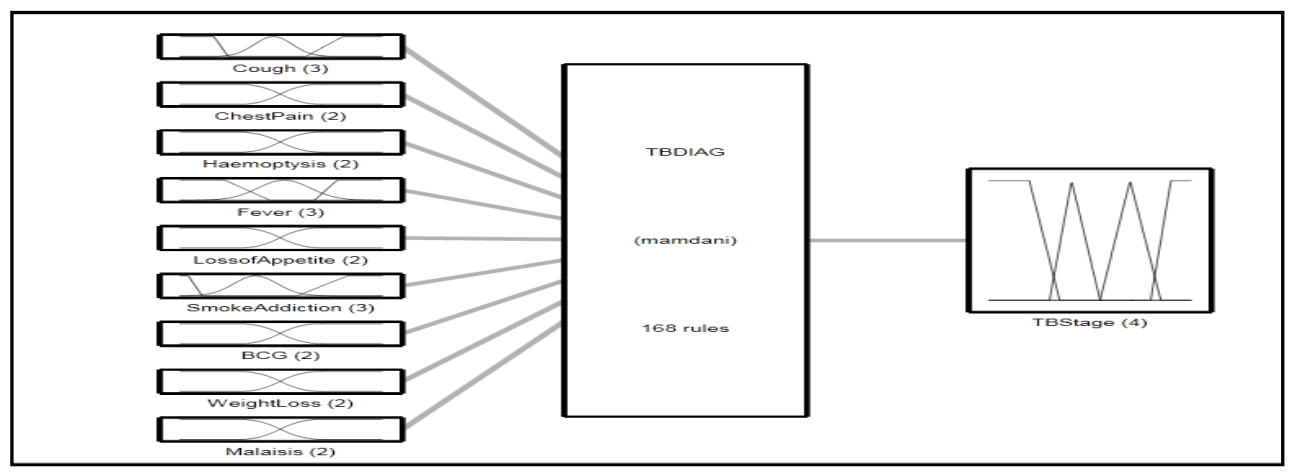

Figure 4. Fuzzy inference System for diagnosis of Tuberculosis

Figure 4 illustrates a total number of input variables taken during diagnosis of tuberculosis. A total number of input attributes is a cough, chest pain, fever, haemoptysis, loss of appetite, smoke addiction, BCG, weight loss and malaises. Each of input consists of two or three triangular or trapezoidal membership functions. Mamdani system is adopted during analysis due to its capability to describe expertise knowledge in more intuitive and similar to a human like operator. Also, Mamdani type systems are capable of handling substantial burden [12]. The output i.e. TBStage is taken in percentage in the range from $0 \%$ to $100 \%$ and consists of four triangular membership functions. A total number of constructed fuzzy rules is 168 that classify each symptom according to the description interviewed by a pulmonary physician.

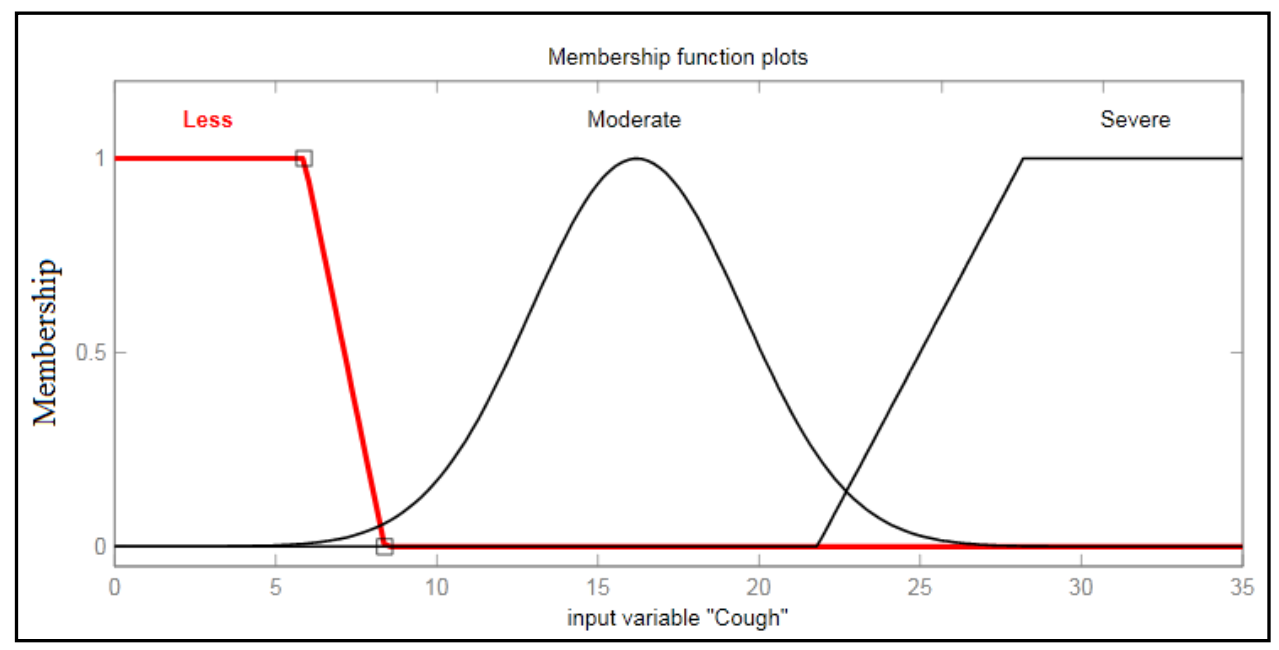

Figure 5. Fine tuned membership plot of Cough 


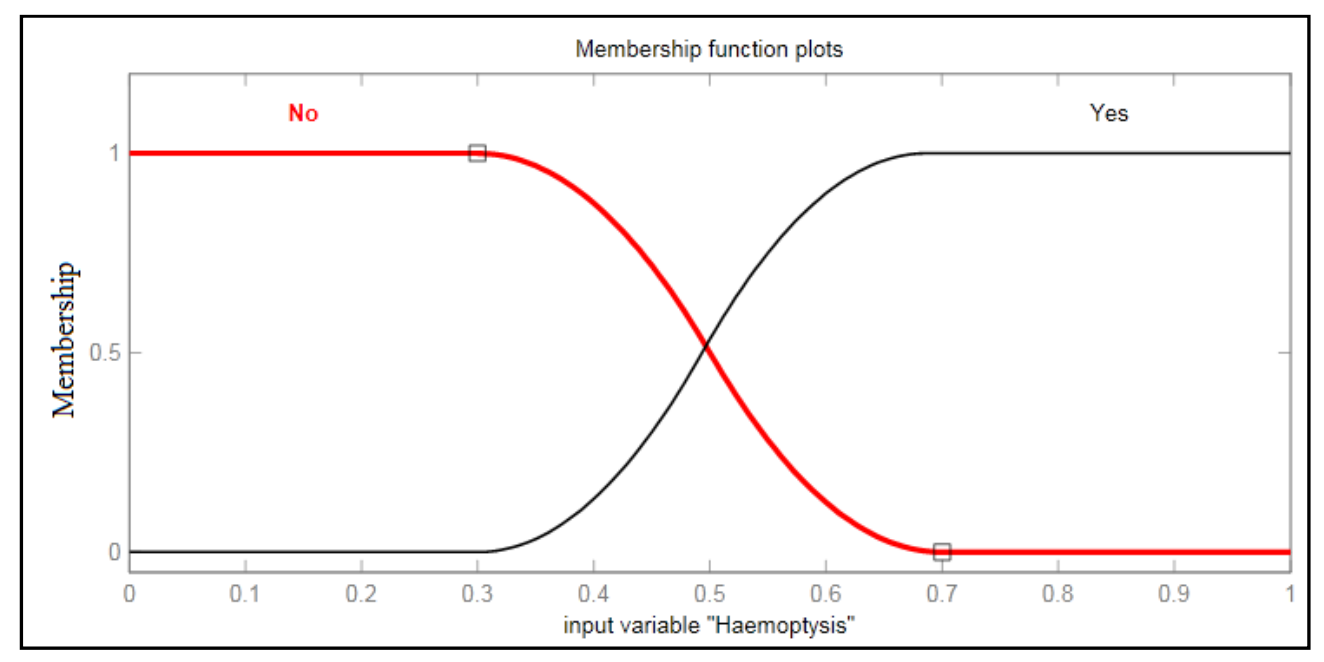

Figure 6. Fine tuned membership plot of Haemoptysis

Figure 5 illustrates membership plot for cough consisting of three membership function values less, moderate and severe. Trapezoidal membership function is employed to represent value less in which cough last for less than a week consisting membership parameter as (-3.17 -1.59 5.88 8.38), gauss membership function used to represent value moderate in which cough last from one to two weeks having membership parameter as $(3.295$ 16.2) trapezoidal membership function is used to represent severe value in which cough lasts from more than four weeks having parameter (21.8 28.1941 .443 .8 ). Figure 6 illustrates membership plot for haemoptysis consisting of two bipolar membership values no and yes. $\mathrm{Z}$ membership function is employed to represent value no having parameter $(0.30 .7), \mathrm{S}$ membership function is employed to represent value yes consisting membership parameter as $(0.30 .6865)$.

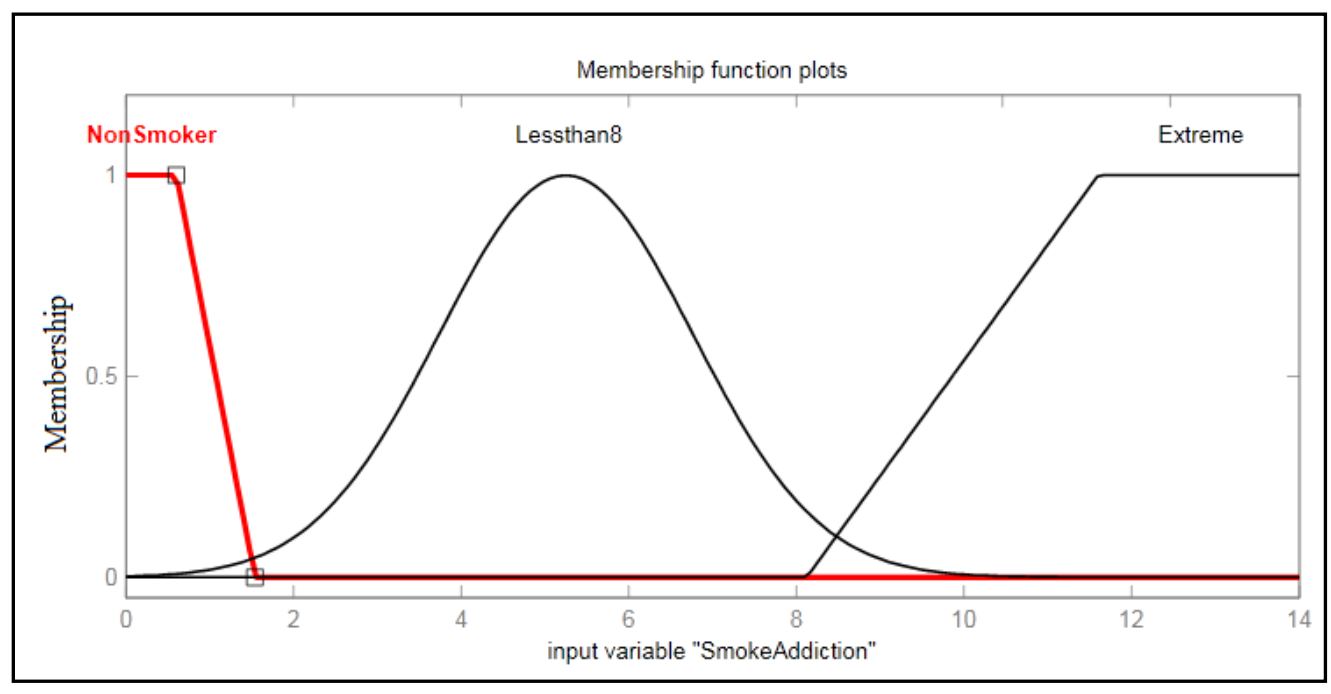

Figure 7. Fine tuned membership plot for Smoke Addiction 
International Journal on Soft Computing (IJSC) Vol.6, No. 3, August 2015

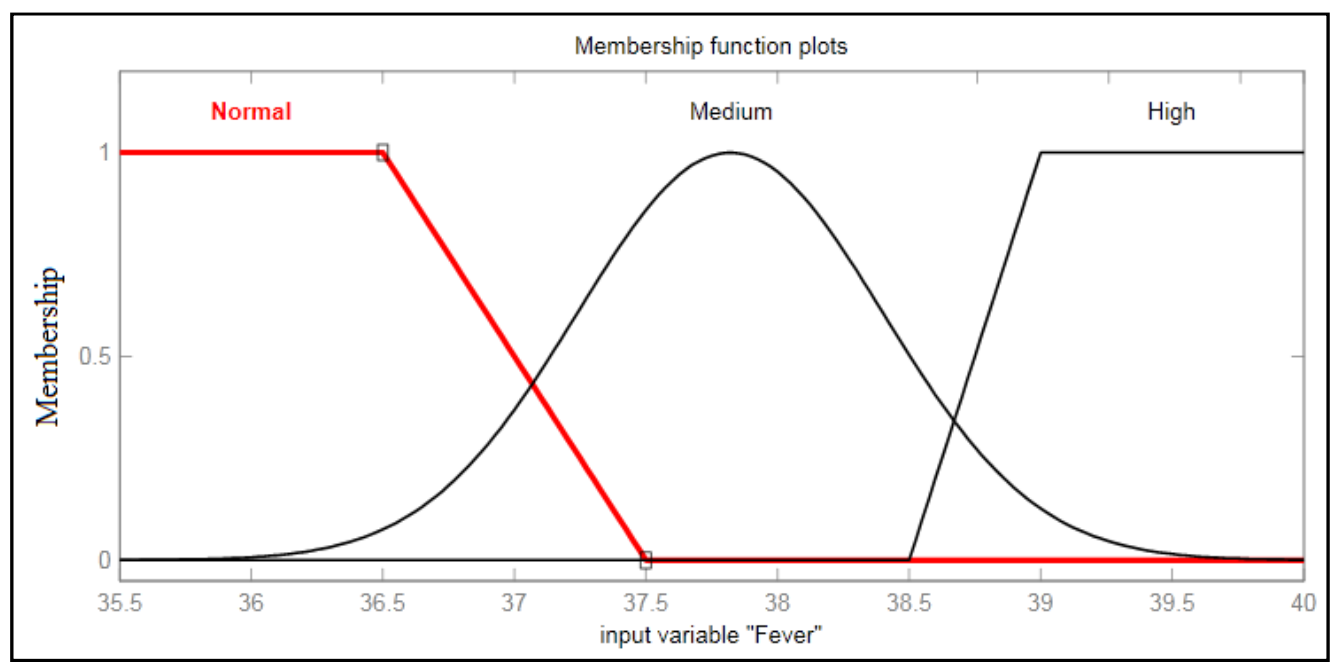

Figure 8. Fine tuned membership plot for Fever

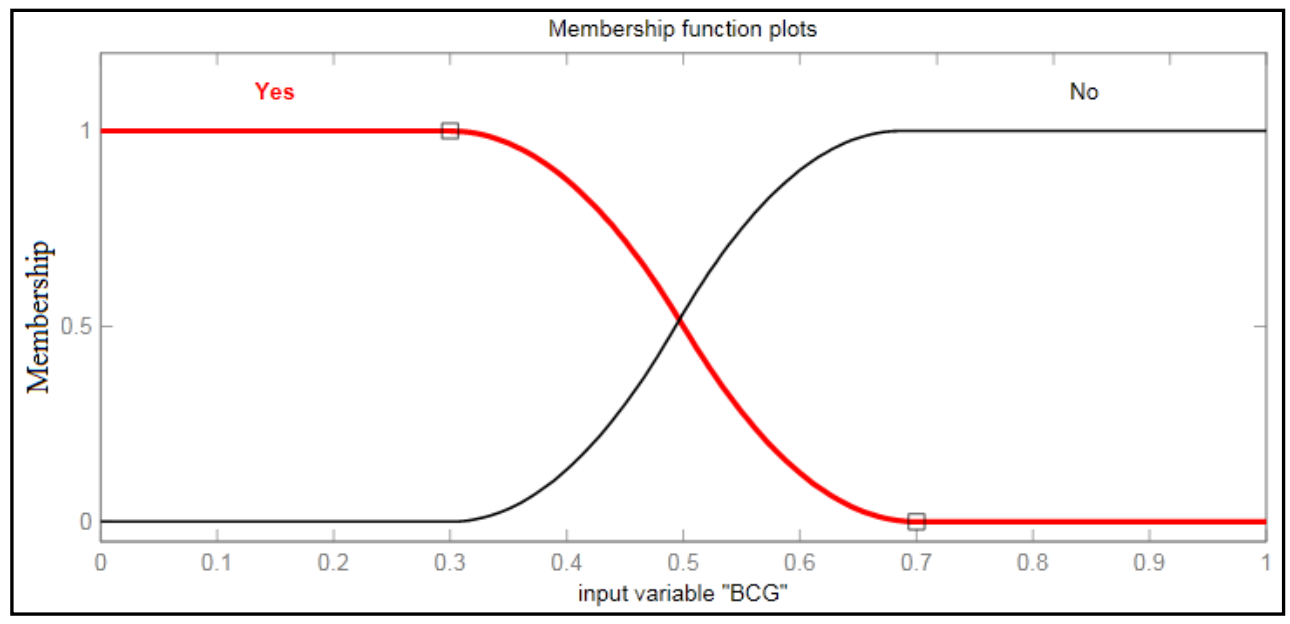

Figure 9. Fine tuned membership plot for BCG vaccine

Figure 7 represent value of smoke addiction in which values of cigarettes consumed by a person per day is represented using three membership function in $(0$ 14). Trapezoidal membership function is employed to represent value non smoker which indicates that either one or none cigarette consumed by a person having parameter as (-2.2 $-20.61 .541)$, gauss membership function used to represent value less than eight cigarettes per day having membership parameter as (1.51 5.25), trapezoidal membership function is used to represent extreme value in cigarettes consumed by person is above eight per day having parameter $\left(\begin{array}{llll}8.122 & 11.6 & 16.6 & 17.5\end{array}\right)$. Figure 8 represent membership function plot for fever in Fahrenheit, range of input for this attribute is (35.5 40). It consists of three membership function values normal, medium, high. Trapezoidal membership function is employed to represent value normal in which fever remain normal having membership parameter as (30 32.536 .537 .5 ), gauss membership function used to represent value moderate in which fever value is above normal having membership parameter as $(0.58137 .82)$, trapezoidal membership function is used to represent high value in which fever is above the normal value having parameter (38.5 394143 ). Figure 9 illustrates membership plot for BCG vaccination consisting of two bipolar membership values no and yes in range $\left(\begin{array}{ll}0 & 1\end{array}\right)$. If vaccination is taken by patient then occurrence of probability infection is reduced to some extend else person 
is at more risk of having infection. $\mathrm{Z}$ membership function is employed to represent value yes having parameter $(0.30 .7)$, s membership function is employed to represent value no consisting membership parameter as $(0.30 .6865)$.

The output stage of tuberculosis is expressed by fuzzy linguistic values such as mild (A1), moderate (A2), severe (A3) and very severe (A4) as shown in figure 10. It consists of four triangular membership function having values mild, moderate, severe and very severe. Trapezoidal membership function is employed to determine stage mild having parameter range ($1-0.50 .20 .35$ ), triangular membership is employed to determine class moderate having parameter range (0.3 0.4090 .55$)$, triangular membership is employed to determine class severe consisting of parameter range $(0.550 .69830 .85)$, trapezoidal membership function is employed to determine class very severe consisting of parameter range (l0.8 0.91 .22$)$.

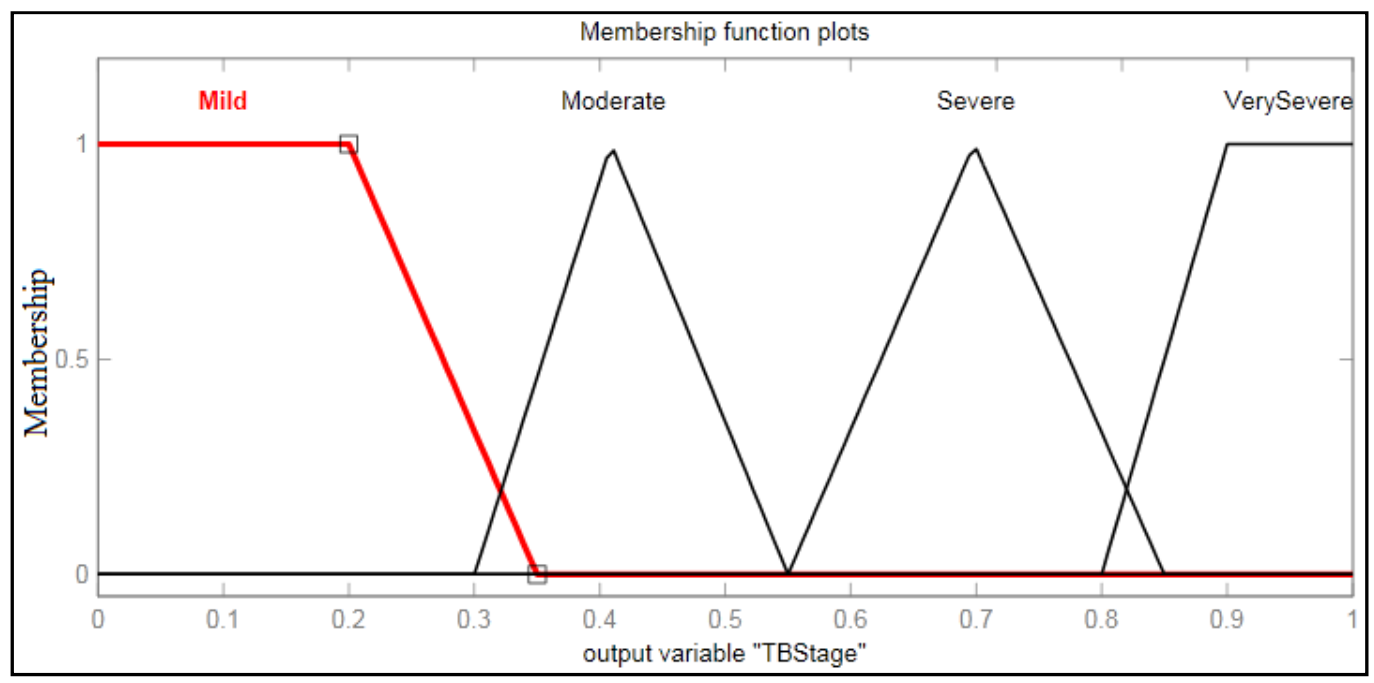

Figure 10. Fine tuned membership plot for Tuberculosis stage

The degree of membership is determined by plugging input parameter into horizontal axis and projecting vertically to upper boundary of membership function [22]. In distinction to fuzzification, defuzzification performs transformation from associate output fuzzy set obtained by inference engine to a numerical value. The foremost standard defuzzification technique is centre method, which implies centre of area under the curve. It can be expressed by using Equation 3 as follows:

$$
X_{d}^{t}=\int \mu c^{\prime}(\mathrm{x}) \cdot x d x / \mu_{\mathrm{c}}{ }^{\prime}(\mathrm{x}) \mathrm{dx}
$$

where $\mu_{\mathrm{c}}{ }^{\prime}(\mathrm{x})$ is the membership function of output fuzzy set $\mathrm{C}^{\prime}$ obtained by inference engine. The value that undergoes defuzzification to compute different output conditions are described using Table 2. 
International Journal on Soft Computing (IJSC) Vol.6, No. 3, August 2015

Table 2. Rulebase for Mamdani fuzzy inference system

\begin{tabular}{|c|c|c|c|c|c|c|c|c|c|c|}
\hline \multirow{2}{*}{$\begin{array}{l}\text { Rul } \\
\text { e } \\
\text { no. }\end{array}$} & \multicolumn{8}{|c|}{ IF } & \multirow{2}{*}{\begin{tabular}{|l|} 
Mala \\
ises
\end{tabular}} & \multirow{2}{*}{$\begin{array}{l}\text { THEN } \\
\text { Output }\end{array}$} \\
\hline & Cough & $\begin{array}{l}\text { Chest } \\
\text { Pain }\end{array}$ & $\begin{array}{l}\text { Haemo } \\
\text { ptysis }\end{array}$ & Fever & $\begin{array}{l}\text { Loss } \\
\text { of } \\
\text { appetit } \\
\mathrm{e}\end{array}$ & $\begin{array}{l}\text { Smoke } \\
\text { addiction }\end{array}$ & $\mathrm{BCG}$ & $\begin{array}{l}\text { Weight } \\
\text { loss }\end{array}$ & & \\
\hline 1. & Less & No & No & Yes & No & Non & No & Yes & Yes & Mild \\
\hline 2. & Less & No & No & Normal & Yes & Less than 8 & No & Yes & Yes & Mild \\
\hline 3. & Less & No & Yes & High & No & Non & Yes & No & Yes & Moderate \\
\hline 4. & Less & No & No & Normal & No & Non & No & Yes & Yes & Mild \\
\hline 5. & Less & No & No & Normal & No & Less than 8 & No & Yes & Yes & Mild \\
\hline 6. & Less & No & No & Medium & No & Less than 8 & No & Yes & Yes & Severe \\
\hline 7. & Severe & Yes & No & Medium & No & Extreme & No & Yes & Yes & V.severe \\
\hline 8. & Less & No & No & High & Yes & Non & Yes & Yes & Yes & Moderate \\
\hline 9. & Less & No & No & High & Yes & Less than 8 & No & Yes & Yes & Severe \\
\hline 10. & Severe & Yes & Yes & High & No & Less than 8 & No & Yes & Yes & V.severe \\
\hline 11. & Less & No & No & High & No & Non & Yes & Yes & Yes & Moderate \\
\hline 12. & Less & No & Yes & Normal & Yes & Extreme & No & Yes & Yes & Severe \\
\hline 21. & Less & No & Yes & Normal & No & Non & No & Yes & Yes & Severe \\
\hline 22 . & Less & No & Yes & Normal & No & Less than 8 & No & Yes & Yes & Severe \\
\hline 23. & Mod. & Yes & No & Normal & Yes & Extreme & No & Yes & Yes & Sev \\
\hline 24. & Less & No & Yes & Medium & No & Less than 8 & No & Yes & Yes & Severe \\
\hline 25. & Less & Yes & No & Normal & No & Less than 8 & No & Yes & Yes & Severe \\
\hline 26. & Mod. & No & No & Normal & No & Extreme & Yes & Yes & Yes & Moderate \\
\hline 27. & Mod. & No & No & Medium & No & Less than 8 & No & Yes & Yes & Severe \\
\hline 28. & Mod. & No & Yes & Normal & No & Non & No & Yes & Yes & Severe \\
\hline 29. & Mod. & Yes & No & Normal & No & Extreme & No & Yes & Yes & Severe \\
\hline 30. & Severe & No & No & Normal & No & Extreme & No & Yes & Yes & Severe \\
\hline 31. & Severe & No & Yes & Medium & No & Less than 8 & No & Yes & Yes & V.severe \\
\hline 32. & Severe & No & No & Medium & Yes & Less than 8 & No & No & No & Mod \\
\hline 33. & Less & No & No & Normal & No & Extreme & No & Yes & Yes & Mild \\
\hline
\end{tabular}

\section{CONCLUSION}

In this paper, we have dealt with tuberculosis. Under this framework, we have presented a fuzzy diagnosability approach for diagnosis of mycobacterium tuberculosis and formalize reasoning. A fuzzy diagnosability function that takes values in the interval $(0,1)$ has been introduced to characterize the diagnosability degree of fuzzy systems. We have formalized the construction of the diagnosers that are used to perform fuzzy diagnosis. In particular, we have proposed a necessary and sufficient condition for diagnosability of tuberculosis using fuzzy expert system and a rule base method for checking diagnosability condition has been given.

The proposed approach enables us to deal with varying degree of input attributes, which may better deal with problem of fuzziness, impreciseness and subjectivity during analysis process. Further, accuracy analysis of system is calculated and observed value is $77 \%$. This system helps medical pulmonary physicians during diagnosis of ailment by reducing time and to make prompt decision. With the results obtained in this paper, a further issue worthy of consideration to other chronic obstructive pulmonary diseases can be done. The designed system can be extended using number of inputs or using clustering techniques.

\section{ACKNOWLEDGEMENTS}

Navneet Walia author wishes to express her sincere gratitude to Harsukhpreet Singh, Assistant Professor, CT Institute of Technology and Research and Mr. Sharad Tiwari, Research Scholar, 
Thapar University, Patiala and anonymous reviewers for their invaluable suggestions that greatly help to improve the quality of paper.

\section{REFERENCES}

[1] K. Rawat, K. Burse, "A Soft Computing Genetic-Neuro fuzzy Approach for Data Mining and Its Application to Medical Diagnosis," International Journal of Engineering and Advanced Technology (IJEAT), ISSN: 2249 - 8958, vol 3, pp. 409- 411, 2013.

[2] A. Yardimci, "Soft computing in medicine," Applied Soft Computing, Elsevier, vol. 9, doi:10.1016/j.asoc.2009.02.003, pp. 1029-1043, 2009.

[3] V. Prasath, N. Lakshmi, M. Nathiya, N. Bharathan, N. P. Neetha, "A Survey on the Applications of Fuzzy Logic in Medical Diagnosis support systems systems decision," International Journal of Scientific \& Engineering Research, ISSN 2229-5518, vol. 4, 2013.

[4] N. Walia, S. K. Tiwari, R. Malhotra, "Design and Identification of Tuberculosis using Fuzzy Based Decision Support System," Advances in Computer Science and Information Technology, ISSN: 23939907, vol. 2, pp. 57-62, 2015.

[5] F. Amato, A. Lopez, E.M Pena-mendez, P. Vanhara, A. Hampl, "Artificial neural networks in Medical diagnosis," Journal of applied biomedicine, doi: 10.2478/v10136-012-0031-x, ISSN 12140287, pp. 47-58, 2013.

[6] R. Malhotra, N. Singh, Y. Singh, "Genetic Algorithms : Concepts, Design for Optimization of Process Controllers," Canadian Center of Science and Education, vol. 4, pp. 39-54, 2011.

[7] R. Malhotra, N. Singh, Y. Singh, "Soft Computing Techniques for process Control Applications," International Journal on Soft Computing, doi: 10.5121/ijsc.2011.2303, vol. 2, pp. 32-44, 2011.

[8] K.Adlassnig, "Fuzzy Set Theory in Medical Diagnosis," IEEE transaction on System, MAN, and Cybernetics, vol. 16, pp. 260-265, 1986.

[9] M.G Forero, F.Sroubek, G.Cristo, "Identification of tuberculosis bacteria based on shape and color," Real time imaging, Elseveir, doi: 10.1016/j.rti.2004.05.007, vol. 10, pp. 251-262, 2004.

[10] N. Walia, S. K. Tiwari, R. Malhotra, "Design and Identification of Tuberculosis using Fuzzy Based Decision Support System," Advances in Computer Science and Information Technology (ACSIT), ISSN: 2393-9915, vol. 2, pp. 57- 62, 2015.

[11] K. Imle, "Automated Detection of Early Lung Cancer and Tuberculosis Based on X- Ray Image Analysis," Proceedings of sixth international conference on Signal, Speech, Image processing, pp. 110-115, 2006.

[12] N. Walia, H. Singh, A. Sharma "ANFIS : Adaptive Neuro-Fuzzy Inference System- A Survey," International Journal of Computer Applications (IJCA), vol. 123, pp. 32-38, 2015.

[13] E.I. Papageorgiou, N.I. Papandrianos, G. Karagianni, G.C. Kyriazopoulos, "A Fuzzy Cognitive Map based tool for prediction of infectious diseases," Transaction on FUZZ-IEEE, pp. 2094-2099, 2009.

[14] U. Dev, A. Sultana, S. Talukder, N.K. Mitra, "A Fuzzy Logic Approach to Decision Support in Medicine," Bangladesh Journal of Scientific and Industrial Research, vol. 46, pp. 41-46, 2011.

[15] P. Srivastava, N. Sharma, "A Spectrum of Soft Computing Model for Medical Diagnosis," Applied Mathematics \& Information Sciences, doi.org/10.12785/amis/080336, vol. 1230, pp. 1225-1230, 2014.

[16] C.Loganathan, K.V.Girija "Hybrid Learning For Adaptive Neuro Fuzzy Inference System," International Journal Of Engineering And Science, ISSN: 2278-4721, vol. 2, no. 11, pp. 6-13, 2013.

[17] O.Tinuke, Y.Sanni, "Dental Expert System," International Journal of Applied Information Systems, ISSN : 2249-0868, vol. 8, pp. 1-15, 2015.

[18] N. Mishra, P. Jha, "Fuzzy expert system and its utility in various field," Recent Research in Science and Technology, ISSN: 2076-5061, vol. 6, pp. 41-45, 2014.

[19] K. Rezaei, R. Hosseini, M. Mazinani, "A fuzzy inference system for assessment of severity of the peptic ulcers," doi: 10.5121/csit.2014.4527, pp. 263-271, 2014.

[20] A. Kaur, A. Kaur, "Comparison of Mamdani-Type and Sugeno-Type Fuzzy Inference Systems for Air Conditioning System," International Journal of Soft Computing and Engineering, ISSN: 2231-2307, vol. 2, pp. 323-325, 2012. 
International Journal on Soft Computing (IJSC) Vol.6, No. 3, August 2015

\section{AUTHORS}

Navneet Walia is pursuing M.TECH final year in department of Electronics and Communication Engineering at CT Institute of Technology and Research, Jalandhar. She has done her B.TECH in trade Electronics and Communication engineering from CEM college of Engineering and Management. She has presented many papers in national and international conferences. Her topic of research is fuzzy logic, neurofuzzy, genetic algorithm and its applicability to industrial sector.

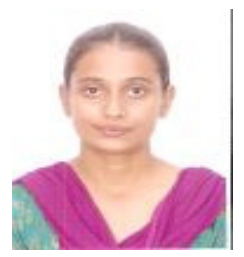

